\title{
Mitteilungen
}

Schweizerische Herzstifung

\section{Forschungspreis 2019}

Die Schweizerische Herzstiftung verleiht jährlich eine mit 20000 CHF dotierte Auszeichnung für eine oder mehrere hervorragende wissenschaftliche Forschungsarbeiten auf dem Gebiet der Prävention, Diagnose und Behandlung der Herz-Kreislauf-Krankheiten.

Teilnahmeberechtigt sind Schweizer Forscherinnen und Forscher oder Forschungsteams, die im In- oder Ausland tätig sind, sowie in der Schweiz arbeitende Ausländer. Der Bewerber darf zum Zeitpunkt der Bewerbung nicht älter als 45-jährig sein. Ehemalige Preisträger und Arbeiten, die bereits ausgezeichnet wurden, können nicht berücksichtigt werden. Die Wahl des Preisträgers erfolgt durch die Kommission Forschung der Schweizerischen Herzstiftung. Weitere Informationen und das detaillierte Reglement finden Sie unter www.swissheart.ch/forschungspreis.

Bewerber reichen bis am 31. Dezember 2018 (Datum des Poststempels) per Post (jeweils 1 Exemplar) sowie zusätzlich in elektronischer Form (Word- oder PDF-File) folgende Unterlagen in englischer Sprache ein:

- unterschriebenes Begleitschreiben

- die zur Beurteilung vorgesehene(n) Arbeit(en) / Publikation(en)
- Literaturverzeichnis

- $\quad$ kurzes Curriculum Vitae (max. 1 Seite)

- $\quad$ kurze Zusammenfassung des Lebenswerkes (2 Seiten)

an:

Schweizerische Herzstiftung

Sekretariat Forschung

Dufourstrasse 30

Postfach 368

3000 Bern 14

Tel. 0313888080

Fax 0313888088

E-Mail: research[at]swissheart.ch www.swissheart.ch/researchprize

\section{Aktuelles Thema auf unserer Website} www.saez.ch/de/tour-dhorizon

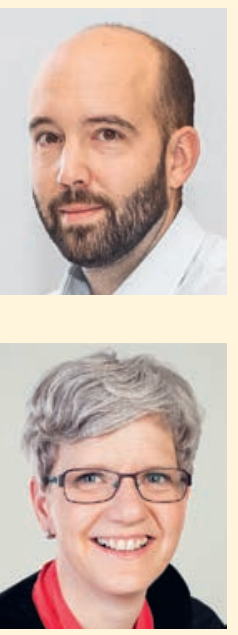

Interview mit Dr. sc. Dominik Glinz, Institut für klinische Epidemiologie und Biostatistik, Universitätsspital Basel

\section{Verbesserungspotential beim Antibiotikaeinsatz}

Eine in der Schweiz durchgeführte, landesweite Studie zeigt, dass es in den Hausarztpraxen durchaus Verbesserungspotential beim Antibiotikaeinsatz gibt.

Sandra Ziegler, Vorsitzende der Geschäftsleitung EMH Schweizerischer Ärzteverlag AG

\section{«Die Qualität ist unsere grösste Stärke»}

Der Schweizerische Ärzteverlag unter neuer Führung. 